\title{
Demonstration of the reproducibility of free-breathing diffusion-weighted MRI and dynamic contrast enhanced MRI in children with solid tumours: a pilot study
}

\author{
Keiko Miyazaki • Neil P. Jerome • David J. Collins • Matthew R. Orton • James A. d'Arcy • \\ Toni Wallace • Lucas Moreno • Andrew D. J. Pearson • Lynley V. Marshall • Fernando Carceller • \\ Martin O. Leach • Stergios Zacharoulis • Dow-Mu Koh
}

Received: 5 September 2014 /Revised: 30 January 2015 / Accepted: 12 February 2015 /Published online: 15 March 2015

(C) The Author(s) 2015. This article is published with open access at Springerlink.com

\begin{abstract}
Objectives The objectives are to examine the reproducibility of functional MR imaging in children with solid tumours using quantitative parameters derived from diffusionweighted (DW-) and dynamic contrast enhanced (DCE-) MRI.

Methods Patients under 16-years-of age with confirmed diagnosis of solid tumours $(n=17)$ underwent freebreathing DW-MRI and DCE-MRI on a $1.5 \mathrm{~T}$ system, repeated 24 hours later. DW-MRI (6 b-values, 0 $1000 \mathrm{sec} / \mathrm{mm}^{2}$ ) enabled monoexponential apparent diffusion coefficient estimation using all $\left(\mathrm{ADC}_{0-1000}\right)$ and
\end{abstract}

K. Miyazaki $\cdot$ N. P. Jerome $\cdot$ D. J. Collins $\cdot$ M. R. Orton $\cdot$

J. A. d'Arcy $\cdot$ M. O. Leach $(\square)$

Cancer Research UK Cancer Imaging Centre at The Institute of

Cancer Research, London SM2 5NG, UK

e-mail: martin.leach@icr.ac.uk

T. Wallace $\cdot$ D.-M. Koh

Department of Radiology, Royal Marsden Hospital,

London, England, UK

L. Moreno • A. D. J. Pearson • L. V. Marshall • F. Carceller •

S. Zacharoulis

Paediatric Drug Development Team, Divisions of Cancer

Therapeutics and Clinical Studies, The Institute of Cancer Research,

London SM2 5NG, UK

L. Moreno

Clinical Research Programme, Spanish National Cancer Research Centre (CNIO), Melchor Fernandez Almagro 3,

28029 Madrid, Spain

L. Moreno · A. D. J. Pearson • L. V. Marshall • F. Carceller •

S. Zacharoulis

Paediatric Drug Development Unit, Children and Young People's

Unit, The Royal Marsden NHS Foundation Trust, Downs Road,

Sutton SM2 5PT, UK only $\geq 100 \mathrm{sec} / \mathrm{mm}^{2}\left(\mathrm{ADC}_{100-1000}\right)$ b-values. DCE-MRI was used to derive the transfer constant $\left(\mathrm{K}^{\text {trans }}\right)$, the efflux constant $\left(\mathrm{k}_{\mathrm{ep}}\right)$, the extracellular extravascular volume $\left(\mathrm{v}_{\mathrm{e}}\right)$, and the plasma fraction $\left(\mathrm{v}_{\mathrm{p}}\right)$, using a study cohort arterial input function (AIF) and the extended Tofts model. Initial area under the gadolinium enhancement curve and pre-contrast $\mathrm{T}_{1}$ were also calculated. Percentage coefficients of variation $(\mathrm{CV})$ of all parameters were calculated.

Results The most reproducible cohort parameters were $\mathrm{ADC}_{100-1000}(\mathrm{CV}=3.26 \%)$, pre-contrast $\mathrm{T}_{1}(\mathrm{CV}=6.21 \%)$, and $\mathrm{K}^{\text {trans }}(\mathrm{CV}=15.23 \%)$. The $\mathrm{ADC}_{100-1000}$ was more reproducible than $\mathrm{ADC}_{0-1000}$, especially extracranially $(\mathrm{CV}=$ $2.40 \%$ vs. $2.78 \%$ ). The AIF ( $n=9)$ derived from this paediatric population exhibited sharper and earlier first-pass and recirculation peaks compared with the literature's adult population average.

Conclusions Free-breathing functional imaging protocols including DW-MRI and DCE-MRI are well-tolerated in children aged 6 - 15 with good to moderate measurement reproducibility.

Key Points

- Diffusion MRI protocol is feasible and well-tolerated in a paediatric oncology population.

- DCE-MRI for pharmacokinetic evaluation is feasible and well tolerated in a paediatric oncology population.

- Paediatric arterial input function (AIF) shows systematic differences from the adult population-average AIF.

- Variation of quantitative parameters from paired functional MRI measurements were within $20 \%$.

Keywords Reproducibility of results · Diffusion magnetic resonance imaging $\cdot$ Paediatrics $\cdot$ Medical oncology .

Functional magnetic resonance imaging 


$\begin{array}{ll}\text { Abbreviations } \\ \text { ADC } & \text { Apparent diffusion coefficient } \\ \text { AIF } & \text { Arterial input function } \\ \text { CI } & \text { Confidence interval } \\ \text { CV } & \text { Coefficient of variation } \\ \text { DCE- } & \text { Dynamic contrast enhanced magnetic resonance } \\ \text { MRI } & \text { imaging } \\ \text { DW-MRI } & \text { Diffusion-weighted magnetic resonance imaging } \\ \text { GRAPPA } & \text { Generalised autocalibrating partially parallel ac- } \\ & \text { quisition acceleration } \\ \text { IAUGC } & \text { Initial area under the gadolinium curve } \\ \text { MRI } & \text { Magnetic resonance imaging } \\ \text { PNET } & \text { Primitive neuroendocrine tumour } \\ \text { ROI } & \text { Region of interest }\end{array}$

\section{Introduction}

Development of anti-cancer therapeutics targeted to specific biological pathways, involved in tumour growth and progression, and inducing cytostasis rather than substantial tumour shrinkage, has led to a need for non-invasive biomarkers reporting on drug action and tumour response beyond simple radiological assessment based on lesion size. Improving survival rates of poor-prognosis childhood cancers via clinical introduction of new molecularly targeted anti-cancer therapeutics, through trials incorporating imaging for proof-oftarget inhibition or predictive biomarkers, is a priority [1-3], and will accelerate and improve the paediatric drug development process.

Diffusion-weighted (DW) and Dynamic ContrastEnhanced (DCE) MRI are imaging-based techniques that can monitor and predict a response to conventional and novel targeted therapeutic agents in clinical trials [4-17]. DW-MRI provides information on tissue cellularity, integrity of cellular membranes, and tortuosity of the extracellular space. Malignant tumours are usually hypercellular compared with their tissues of origin, and, thus, show a low apparent diffusion coefficient (ADC). DCE-MRI enables the assessment of parameters pertinent to the state of tumour vasculature by tracking the kinetics of an intravenously administered contrast agent. DCE-MRI measurements can be obtained from parameters such as: the volume transfer constant between plasma and extracellular space $\left(\mathrm{K}^{\text {trans }}\right)$, the efflux rate constant between extracellular extravascular space and plasma $\left(\mathrm{k}_{\mathrm{ep}}\right)$, the fractional volume of extracellular extravascular space per unit volume of tissue $\left(\mathrm{v}_{\mathrm{e}}\right)$, the fractional volume of blood plasma per unit volume of tissue $\left(\mathrm{v}_{\mathrm{p}}\right)$, the initial area under the gadolinium curve over 60 seconds after arrival of contrast agent
(IAUGC60), and the longitudinal relaxation time before administration of contrast agent (pre-contrast $\mathrm{T}_{1}$ ).

While these functional imaging biomarkers have been extensively used in early clinical trials in the adult setting, there is very little information in the paediatric population where, due to the differences in vascular physiology and biology of paediatric tumours, they may be different. There are also important practical issues that make functional measurements more challenging in children, including logistical difficulties, movement during the procedure, and difficulties obtaining intravenous access. Additionally, the rarity of the conditions necessitate multicentre clinical trials, requiring additional validation and quality assurance procedures. For a prospective study of parameter reproducibility - rather than parameter values - it is possible to include a diverse range of pathologies, giving increased recruitment within a cohort where cancer is rare, and increased generality of the reproducibility assessment. Improved knowledge of reproducibility for functional imaging will guide further development and incorporation as exploratory or secondary endpoints in multi-centre phase I/II clinical trials of cancer therapeutics in children, particularly those that have an antiangiogenic effect. Specifically, DCE-MRI and DW-MRI may demonstrate proof-of-mechanism that anti-angiogenics cause changes in tumour vasculature and tumour cellularity. In addition, tumour imaging characteristics at study entry or changes in tumour imaging characteristics early in therapy may be predictive biomarkers for objective response and clinical benefit.

The purpose of this study is to examine the feasibility of performing functional MRI in children with solid tumours by evaluating the reproducibility of quantitative parameters derived from diffusion-weighted (DW-) and dynamic contrast enhanced (DCE-) MRI.

\section{Materials and methods}

Patient population

This prospective study was approved by the institutional review board; prior written consent was obtained from each child's parent/legal guardian, with assent from each child. Inclusion criteria were: patients (a) less than 16 years old, (b) with confirmed diagnosis of a solid tumour, (c) with a measurable target lesion ( $\geq 2 \mathrm{~cm}$ ), and (d) requiring MRI as part of their routine care. Exclusion criteria were patients with: (a) general anaesthesia required for their scans, (b) renal function impairment, (c) previous allergy to contrast, or contraindications to MRI, (d) lung metastases only, and (e) disease sites that may result in substantial artefacts on the DCE-MRI and DW-MRI studies. 


\section{MRI studies}

Imaging was performed with a $1.5 \mathrm{~T}$ Magnetom Avanto system (Siemens Healthcare, Erlangen, Germany) using a phased-array head coil (intra-cranial studies) or a phasedarray body coil (extra-cranial studies). DW- and DCE-MRI was performed during the initial routine MRI, and repeated after 24 hours.

\section{Diffusion-weighted MRI}

The free-breathing DW-MRI protocol used a multi-slice single-shot echo-planar imaging sequence with: echo time, $75 \mathrm{msec}$; repetition time, $3500 \mathrm{msec}$; matrix, $128 \times 128$ (interpolated to $256 \times 256$ ); 24 slices with $5 \mathrm{~mm}$ thickness; generalised autocalibrating partially parallel acquisition acceleration (GRAPPA) factor of two; spectral adiabatic inversion-recovery fat suppression, and three signal averages. Six diffusion gradients (b-values: 0, 50, 100, 300, 600, and $1000 \mathrm{sec} /$ $\mathrm{mm}^{2}$ ) were applied in three orthogonal directions and averaged to provide isotropic trace-weighted images. Total time for DW-MRI was 3 min 30 seconds. The fields of view were $220 \times 220 \mathrm{~mm}^{2}$ in the transverse orientation (intra-cranial studies) and $300 \times 300 \mathrm{~mm}^{2}$ in the coronal orientation (extra-cranial studies). The central imaging slice was positioned through the centre of the tumour.

\section{Dynamic contrast-enhanced MRI}

The free-breathing DCE-MRI protocol consisted of the following:

i.) A proton density-weighted three-dimensional spoiled gradient-echo sequence with: echo time, $0.95 \mathrm{msec}$; repetition time, $3.00 \mathrm{msec}$; matrix, $128 \times 128$ (interpolated to $256 \times 256$ ); 14 partitions with $5 \mathrm{~mm}$ thickness; GRAPPA factor of two; flip angle, $3^{\circ}$; and ten signal averages. The DCE-MRI volume was centred on the DW-MRI volume.

ii.) A dynamic $\mathrm{T}_{1}$-weighted acquisition with identical parameters, except for a flip angle of $16^{\circ}$, and one signal average. Eighty volumes at 3.23 seconds each were acquired in approximately 4 minutes. At the fifth volumetric acquisition, gadolinium-based contrast agent (single dose, $0.1 \mathrm{mmol} / \mathrm{kg}$ ) was injected followed by $10 \mathrm{ml}$ of saline. Magnevist ${ }^{\circledR}$ was the contrast agent used initially, but it was changed to Dotarem ${ }^{\circledR}$ following a change in local policy regarding MR contrast agents. In children without an implanted central line, injections were performed using a power injector through a peripherally-inserted intravenous cannula. The flow rate varied between $1-3 \mathrm{ml} / \mathrm{sec}$ appropriate to the cannula. A number $(n=4)$ of patients had a Hickman-type central line in place; to avoid an additional invasive procedure in these children, contrast was delivered manually into the central line via a three-way tap by an expert radiologist (D.M.K).

iii.) The sequence in i.) was repeated following the dynamic acquisition, to allow subsequent conversion of signal intensity to contrast agent concentration $[18,19]$. Total time for DCE-MRI was 6 minutes.

Patients were imaged twice, 24 hours apart without treatment intervention, using the same imaging and injection protocols.

\section{Image analysis}

Images were assessed and regions-of-interest (ROIs) were drawn by an expert radiologist (D.M.K); ROIs were drawn around the tumour for three central slices of the imaging volume, and in the matching slices of the repeat study. Data processing to derive functional parameters was performed offline by a postdoctoral research fellow experienced (6 years) in DW- and DCE-MRI studies. DW-MRI analysis was performed offline using ADEPT software (The Institute of Cancer Research, London, UK). Apparent diffusion coefficient values (ADC, $10^{-6} \mathrm{~mm}^{2} / \mathrm{sec}$ ) were calculated using Levenberg-Marquardt mono-exponential fitting with signal intensities from: (i) all b-values $(0,50,100,300,600$, and $1000 \mathrm{sec} / \mathrm{mm}^{2},{ }^{\prime} \mathrm{ADC}_{0-1000}$ ') and (ii) b-values $\geq 100 \mathrm{sec} /$ $\mathrm{mm}^{2}\left(100,300,600\right.$, and $\left.1000 \mathrm{sec} / \mathrm{mm}^{2},{ }^{\prime} \mathrm{ADC}_{100-1000}\right)$ on a voxel-by-voxel basis.

DCE-MRI data processing was performed off-line using MRIW software (The Institute of Cancer Research, London, UK), using the same slices and ROIs as for the DW-MRI. The proton-density weighted images acquired post-contrast were used with the dynamically acquired $\mathrm{T}_{1}$-weighted images to generate contrast agent concentrations on a per-voxel basis $[18,19]$. Relaxivities of Magnevist ${ }^{\circledR}$ and Dotarem $^{\circledR}$ at $1.5 \mathrm{~T}$ were taken to be 4.3 and $4.2 \mathrm{mM}^{-1} \mathrm{sec}^{-1}$, respectively. The model-independent parameters evaluated were: (i) IAUGC60, in mM.sec, and (ii) the pre-contrast $\mathrm{T}_{1}$, in seconds. Pharmacokinetic model analysis was performed using an extended Tofts model and a population-averaged arterial input function (AIF), measured from a subset of the study cohort where a suitable ROI could be drawn along the descending aorta on or near the central partition (Fig. 1a). Dynamic signal intensities averaged over the ROI were converted to contrast agent concentrations as for the lesion data, and an empirical input function model consisting of components describing the first 


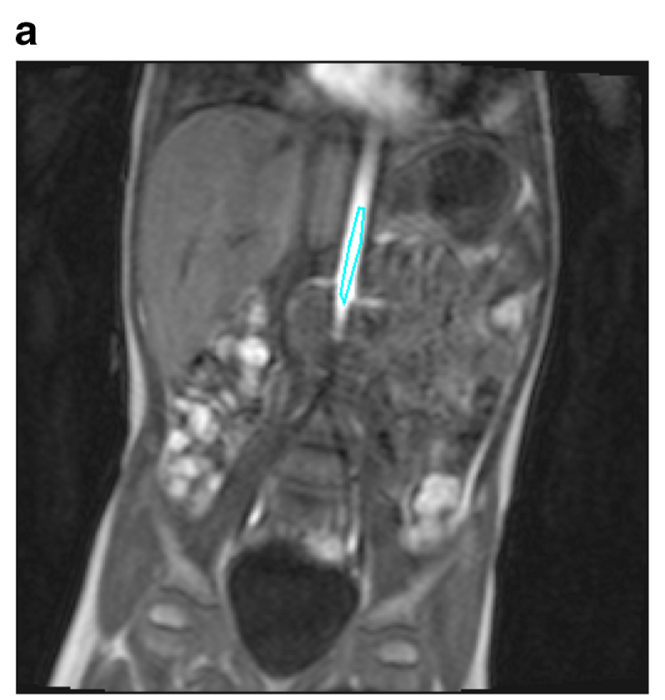

Fig. 1 (a) $T_{1}$-weighted DCE-MR image at peak arterial enhancement in the descending aorta with a region-of-interest (turquoise) used for measurement of arterial input function overlaid. (b) Populationaveraged input functions obtained from a subset of children in this

pass, second pass, and equilibrium phases was fitted to the data $[20,21]$ :

$C_{p}(t)=C_{B}(t) \otimes R_{M}(t)+C_{R}\left(t-t_{R}\right)+C_{R}\left(t-t_{R}\right) \otimes R_{E}(t)$,

where $C_{B}(t)$ models dispersion in the lungs, $C_{R}(t)$ describes the recirculation phase with a delay $t_{R}$, and both are modelled with raised cosine functions [22]. $R_{M}(t)$ is an impulse response modelling the mixing through the heart, given by $R_{M}(t)=\alpha_{M}$ $\exp \left(-\mu_{M} t\right)$, and $R_{E}(t)$ is an impulse response modelling reflux of contrast from the whole-body extra-vascular space: $R_{E}(t)=$ $\alpha_{E} \exp \left(-\mu_{E} t\right)$. The input function is thus defined by eight parameters: $a_{B}, \mu_{B}, \mu_{M}$ (first pass phase), $a_{R}, \mu_{R}, t_{R}$ (second pass phase), $a_{E}$ and $\mu_{E}$ (equilibrium phase). A population-averaged paediatric AIF was constructed by taking the median of the individual AIF parameters after accounting for variations in haematocrit measured in individual patients. The DCE-MRI model-dependent parameters evaluated were: (i) $\mathrm{K}^{\text {trans }}$, in min $^{-}$ ${ }^{1}$, (ii) $\mathrm{k}_{\mathrm{ep}}$, in $\min ^{-1}$, (iii) $\mathrm{v}_{\mathrm{e}}$, and (iv) $\mathrm{v}_{\mathrm{p}}$.

Statistical analysis

For each parameter, the results from voxels in the three analysed slices were combined, and the median parameter value was used in analysis of reproducibility. For modelderived DCE-MRI parameters, values from non-enhancing voxels (where post-onset signal intensities stay within one standard deviation of the pre-contrast mean signal intensity) were not included. The time required for post-processing (performed offline on a laptop, $2.4 \mathrm{GHz}$ processor, 8 GB RAM) varied directly with the size of the tumour ROIs, from approximately 20 seconds to 14 minutes (DCE-MRI) and 10 seconds

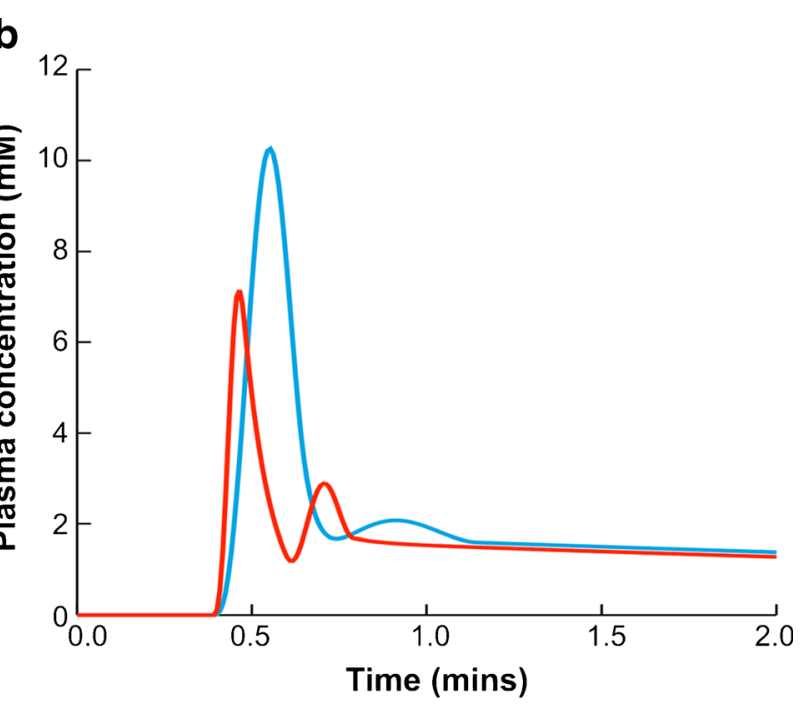

study cohort (red) and from adult patients (blue) [20] fitted to an empirical input function model C. The input function parameters are summarised in Table 1. Only the first two minutes of the concentrationtime course data have been shown for display purposes

to 6.5 minutes (DW-MRI) for the smallest and largest tumours, respectively. Baseline variability between repeat median parameters was assessed by calculating the coefficient of variation $(\mathrm{CV})$ and $95 \%$ confidence interval $(\mathrm{CI})$ limits using log-transformed measurement values for: (i) intra-cranial, (ii) extra-cranial, and (iii) combined cohorts.

\section{Results}

The study cohort comprised 17 patients with solid tumours (eight intracranial, nine extracranial tumours) recruited between January 2010 and August 2013. Median patient age was 11 years (range 6-15 years). Patient characteristics and primary tumour classification are summarised in Table 1.

All patients were cooperative and tolerated the freebreathing imaging protocol well. One patient did not return for a repeat scan owing to logistical difficulties. DW-MRI data in one patient suffered technical failure. Tumours in four patients did not exhibit contrast agent uptake (intracranial: anaplastic astrocytoma and thalamic high-grade glioma; and extracranial: intra-abdominal PNET and neuroblastoma stage 4). Reproducibility values of the DW-MRI parameters were, thus, analysed using 15 pairs of measurements (seven intracranial, eight extracranial), and that of DCE-MRI parameters using 12 pairs of measurements (five intracranial, seven extracranial).

Nine study-specific, individual AIFs were obtained from DCE-MRI. The cohort-averaged paediatric AIF is shown in Fig. $1 \mathrm{~b}$ together with an adult population-averaged AIF refitted to the input function model in Eq. $1[20,23]$ with an assumed haematocrit of 0.42 . First-pass and recirculation peaks were found to be sharper and to occur earlier in the 
Table 1 Patient characteristics and primary tumour classification

\begin{tabular}{|c|c|c|}
\hline & Sex/Age & Pathology \\
\hline \multicolumn{3}{|c|}{ Intracranial tumour } \\
\hline 1 & $\mathrm{~F} / 8$ & Anaplastic astrocytoma \\
\hline 2 & $\mathrm{M} / 7$ & Primitive neuroectodermal tumour \\
\hline 3 & $\mathrm{M} / 10$ & Glioblastoma multiforme \\
\hline 4 & $\mathrm{M} / 11$ & High grade glioma \\
\hline 5 & $\mathrm{~F} / 9$ & Glioblastoma multiforme \\
\hline 6 & $\mathrm{M} / 8$ & Glioblastoma multiforme \\
\hline 7 & $\mathrm{M} / 15$ & Anaplastic astrocytoma \\
\hline 8 & $\mathrm{M} / 11$ & Astrocytoma \\
\hline \multicolumn{3}{|c|}{ Extracranial tumour } \\
\hline 9 & $\mathrm{M} / 7$ & Ganglioneuroblastoma \\
\hline 10 & $\mathrm{M} / 13$ & Spindle cell sarcoma \\
\hline 11 & $\mathrm{~F} / 12$ & Ganglioneuroblastoma \\
\hline 12 & $\mathrm{M} / 14$ & Rhabdomyosarcoma \\
\hline 13 & $\mathrm{M} / 15$ & Sacral myxopapillary ependymoma \\
\hline 14 & $\mathrm{M} / 12$ & Intra-abdominal primitive neuroectodermal tumour \\
\hline 15 & $\mathrm{M} / 6$ & Neuroblastoma \\
\hline 16 & $\mathrm{M} / 13$ & Rhabdomyosarcoma \\
\hline 17 & $\mathrm{M} / 14$ & Neuroendocrine tumour \\
\hline
\end{tabular}

paediatric AIF; parameters for the two population-averaged AIFs are given in Table 2.

The coefficients of variation and $95 \% \mathrm{CI}$ limits for all imaging parameters are summarised in Table 3. The most reproducible DW-MRI and DCE-MRI parameters for the full cohort were $\mathrm{ADC}_{100-1000}(\mathrm{CV}=3.26 \%)$, pre-contrast $\mathrm{T}_{1}$ $(\mathrm{CV}=6.21 \%)$, and $\mathrm{K}^{\text {trans }}(\mathrm{CV}=15.23 \%)$. The $\mathrm{ADC}_{100-1000}$ was found to be more reproducible than $\mathrm{ADC}_{0-1000}$, especially extracranially (CV=2.40\% vs. $2.78 \%$ ).

Example ADC maps and mono-exponential curve fits for $\mathrm{ADC}_{0-1000}$ and $\mathrm{ADC}_{100-1000}$ from intracranial and extracranial tumour patients are shown in Figs. 2 and 3, respectively. The IAUGC60 and $\mathrm{K}^{\text {trans }}$ maps and contrast agent uptake curves (same patients) are shown in Figs. 4 and 5.

Table 2 Summary of input function model parameters [21] for the population-averaged adult and paediatric arterial input functions

\begin{tabular}{llll}
\hline Input function parameters & & Adult & Paediatric \\
\hline First-pass parameters & $\mathrm{a}_{\mathrm{B}}(\mathrm{mM})$ & 60.7 & 86.5 \\
& $\mu_{\mathrm{B}}\left(\mathrm{min}^{-1}\right)$ & 24.8 & 65.9 \\
& $\mu_{\mathrm{M}}\left(\mathrm{min}^{-1}\right)$ & 23.4 & 13.3 \\
Second-pass parameters & $\mathrm{a}_{\mathrm{R}}(\mathrm{mM})$ & 4.96 & 8.19 \\
& $\mu_{\mathrm{R}}\left(\mathrm{min}^{-1}\right)$ & 9.48 & 32.0 \\
& $\mathrm{t}_{\mathrm{R}}\left(\mathrm{min}^{2}\right)$ & 0.0943 & 0.209 \\
Equilibrium phase parameters & $\mathrm{a}_{\mathrm{E}}(\mathrm{mM})$ & 25.4 & 84.3 \\
& $\mu_{\mathrm{E}}\left(\mathrm{min}^{-1}\right)$ & 0.169 & 0.179 \\
\hline
\end{tabular}

\section{Discussion}

Functional imaging has the potential to provide imaging biomarkers for proof-of-mechanism for angiogenesis inhibitors, and to identify those patients more likely to respond to a given therapy, as well as providing more physiological sensitivity when assessing post-treatment response.

The range and specificity of available functional imaging modalities allow improved characterisation of spatial differences in biological properties both within and between tumours, though at the cost of lengthened imaging studies. Paediatric MRI is particularly challenging, since children may not lie still during the entire imaging protocol, find the noise and confined space distressing, or may be unable to cooperate with breath-holding instructions intended to minimise abdominal motion artefacts as used with adults.

Despite the challenges involved, there is increasing use of functional imaging in children with intracranial tumours, as summarised by Peet et al. [24]. There are, however, still very few reports of functional imaging studies in children with extracranial tumours. Despite this paucity, there are studies that report the potential of DW- and DCE-MRI biomarkers for disease characterisation $[25,26]$ and response assessment in various paediatric oncological settings. RodriguezGutierrez et al., evaluated serial DW-MRI to predict treatment response in high-grade paediatric brain tumours and found that responders exhibited a higher regional ADC increase [27]. Guo et al., performed DCE-MRI in children with newly diagnosed, non-metastatic osteosarcoma treated on a phase 2 trial (NCT001456390) [28], finding DCE-MRI derived parameters were potential early prognostic factors for eventfree and overall survival. Recently, Navid et al., published the results of a phase I and clinical pharmacology study of bevacizumab, sorafenib, and low-dose cyclophosphamide in children and young adults with refractory/recurrent extracranial solid tumours [29]; both groups of study patients exhibited a rapid decrease in $\mathrm{K}^{\text {trans }}$ and $\mathrm{v}_{\mathrm{p}}$ in the first 7 days after treatment initiation. Such results demonstrate the emerging roles of functional MR imaging markers for assessing and predicting treatment response in children with cancer, and their potential contribution to the development of stratified treatment regimens.

Baseline reproducibility values of the functional MR parameters are important measures of consistency and reliability that should be evaluated. Several studies have investigated the reproducibility of DW- and DCE-MRI derived parameters in adults $[10,12,30]$; given the added challenges when imaging children, arising from both logistical and physiological differences, reproducibility should be investigated specifically for this cohort.

In this present study functional MR imaging, repeated following 24 hours without treatment intervention, was performed in a paediatric cohort ( 6 to 15 years-of-age) to assess 
Table 3 Reproducibility values of DW-MR imaging and DCE-MR imaging parameters

\begin{tabular}{lccc}
\hline & \multicolumn{1}{c}{ Coefficient of Variation $(\%), 95 \%$ confidence interval limits in parentheses } \\
\hline DW-MRI parameters & Intra-cranial $(n=7)$ & Extra-cranial $(n=8)$ & Intra- and extra-cranial $(n=15)$ \\
ADC $_{0-1000}$ & $3.92(-7.4,8.0)$ & $2.78(-5.3,5.6)$ & $3.74(-7.1,7.6)$ \\
ADC $_{100-1000}$ & $4.09(-7.7,8.4)$ & $2.40(-4.6,4.8)$ & $3.26(-6.2,6.6)$ \\
DCE-MRI parameters & Intracranial $(n=5)$ & Extracranial $(n=7)$ & Intracranial and extracranial $(n=12)$ \\
Pre-contrast $\mathrm{T}_{1}$ & $7.99(-14.5,16.9)$ & $5.19(-9.7,10.7)$ & $6.21(-11.5,12.9)$ \\
IAUGC60 & $9.38(-16.8,20.1)$ & $11.71(-20.5,25.7)$ & $12.77(-22.1,28.3)$ \\
$\mathrm{K}^{\text {trans }}$ & $13.58(-23.3,30.3)$ & $16.79(-27.9,38.7)$ & $15.23(-25.7,34.5)$ \\
$\mathrm{k}_{\mathrm{ep}}$ & $21.74(-34.4,52.4)$ & $19.74(-31.8,46.7)$ & $19.60(-31.7,46.3)$ \\
$\mathrm{V}_{\mathrm{e}}$ & $20.05(-32.2,47.6)$ & $17.48(-28.8,40.5)$ & $18.56(-30.3,43.4)$ \\
$\mathrm{V}_{\mathrm{p}}$ & $50.73(-60.9,155.5)$ & $16.29(-27.2,37.3)$ & $36.24(-49.8,99.1)$ \\
\hline
\end{tabular}

the reproducibility of the derived parameters. All seventeen children who participated in the study were able to cooperate throughout the entire imaging protocol, including injections of contrast agent and saline flush. The quality of data obtained allowed DW-MRI and DCE-MRI model fitting on a voxel-byvoxel basis to generate parametric maps, with parameter reproducibility values comparable to those achieved in adult cohorts $[12,30]$. The diversity of tumours present in the cohort adds to the generality of the result, with both extracranial and intracranial tumour subgroups presenting similar coefficients of variation.

Whole cohort ADC reproducibility was found to be better when excluding the two lowest $b$-values in the mono-exponential fitting procedure, compared to using all b-values; this difference was also present for the extracranial tumour cohort, suggesting that fitting a mono-exponential model for all b-values results in ADC measures that will be biased by the lower b- value data points. The curve fits shown in Figs. 2 and 3 clearly illustrate this bias. The use of a bi-exponential model with "slow" and "fast" diffusion components arising from an optimised diffusion protocol may be more informative, as well as better reflecting the properties of specific tumour types, and should be explored.

For DCE-MRI measurements, we employed a 3-D volumetric protocol with good spatial and temporal resolutions. The dynamic temporal resolution employed $(\sim 3 \mathrm{~s})$ not only helped to minimise motion artefacts but also helped to capture the contrast uptake curves in the tumour and the descending aorta (where signal changes occur rapidly). Using the contrast uptake curves from the descending aorta, it was possible to construct a paediatric population AIF (Fig. 1b), observed to have features distinct from those seen in an adult population [23] and consistent with the relatively high rate of circulation seen in children. Although limited patient numbers preclude investigation of AIF variations with age in this present study, a

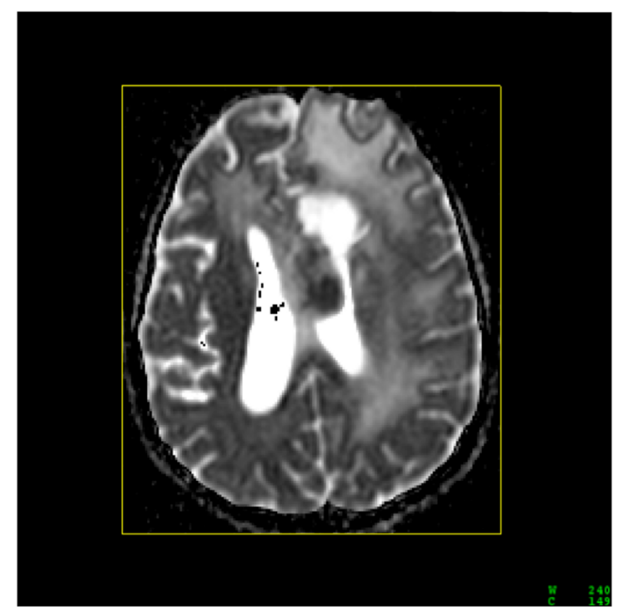

b
Fig. 2 (a) $\mathrm{ADC}_{100-1000}$ map from a 10-year-old boy with glioblastoma multiforme in the left frontal lobe. (b) Example mono-exponential curve fits using signal intensities at $b=0-1000 \mathrm{sec} / \mathrm{mm}^{2}$ (blue) and $b=100-$

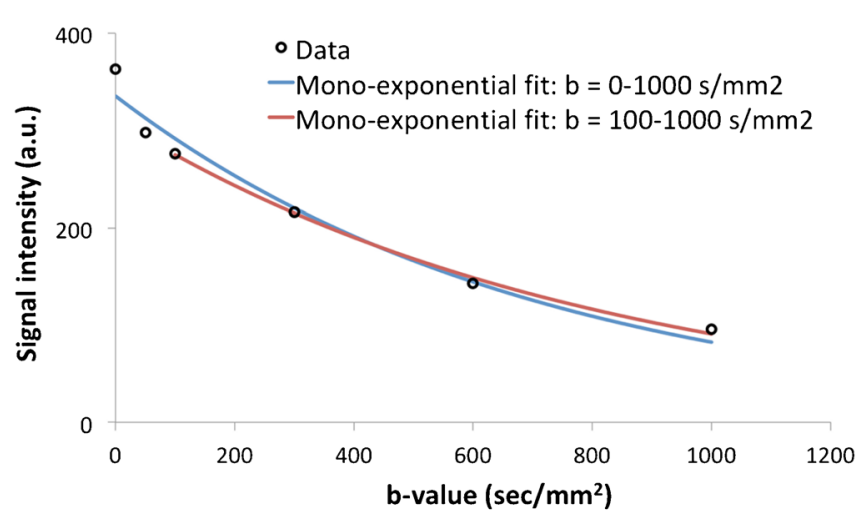

$1000 \mathrm{sec} / \mathrm{mm}^{2}$ (red) from a single pixel in the tumour. The calculated ADC value for the two fits were $1402 \times 10^{-6} \mathrm{~mm}^{2} / \mathrm{sec}$ and $1228 \times 10^{-}$ ${ }^{6} \mathrm{~mm}^{2} / \mathrm{sec}$, respectively 
a

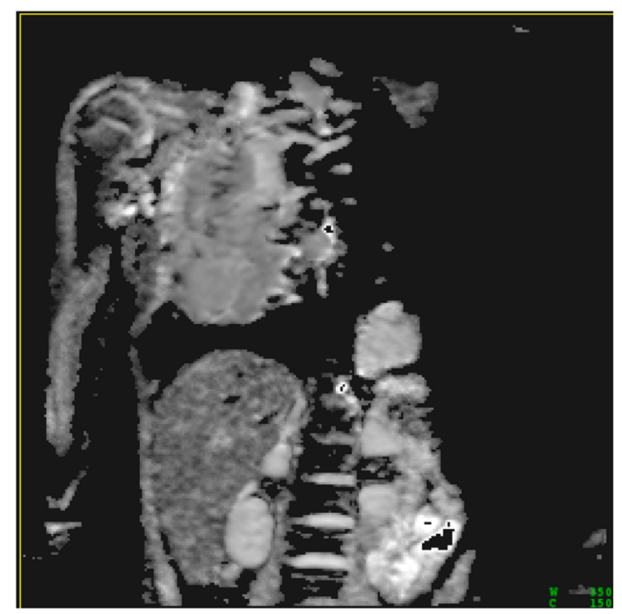

Fig. 3 (a) $\mathrm{ADC}_{100-1000}$ map from a 13-year-old boy with a low grade spindle cell sarcoma attached to the right chest wall. The signal void seen in the top left region is due to a saturation band placed over the heart to avoid propagation of artefacts due to cardiac motion. (b) Example mono-

the differences observed between the paediatric and adult population AIFs highlight the importance of conducting such investigations in the future. Additionally, it is important to optimise the injection protocol in patients with an implanted central venous line to minimise the variability observed with hand injections [20].

Pre-contrast $T_{1}$ was found to be a robust parameter with the best reproducibility $(\mathrm{CV}=6.21 \%)$. This parameter is used in the conversion of signal intensity to contrast agent concentration for quantitative DCE-MRI data analysis, but it is also a potential marker for treatment response $[31,32]$. There is a consensus that IAUGC60 and $\mathrm{K}^{\text {trans }}$ should be used as quantitative endpoints for DCE-MRI when used in the development of anti-angiogenic and anti-vascular agents [33, 34], with a recent report on DCE-MRI quantification supporting a $\mathrm{CV}$ of less than $20 \%$ for these parameters [35]. In our b

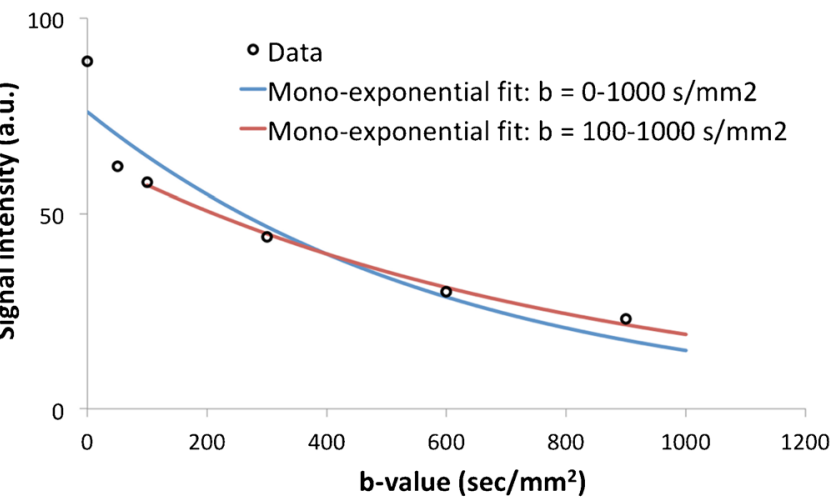

exponential curve fits using signal intensities at $b=0-1000 \mathrm{sec} / \mathrm{mm}^{2}$ (blue) and $b=100-1000 \mathrm{sec} / \mathrm{mm}^{2}$ (red) from a single pixel in the tumour. The calculated ADC value for the two fits were $1626 \times 10^{-}$ ${ }^{6} \mathrm{~mm}^{2} / \mathrm{sec}$ and $1221 \times 10^{-6} \mathrm{~mm}^{2} / \mathrm{sec}$, respectively

study, the CV of IAUGC60 and $\mathrm{K}^{\text {trans }}$ for the whole cohort were $12.77 \%$ and $15.23 \%$, respectively. The reproducibility of other DCE-MRI derived parameters ranged between 12.8 to $19.6 \%$ except for $\mathrm{v}_{\mathrm{p}}$ for the whole cohort $(36.24 \%)$. It is interesting that the DCEMRI parameters generally expressed a higher variability than those from DW-MRI, which may reflect either additional complexities of administering exogenous contrast or, given that the most reproducible DCE-MRI parameters were model-independent, the inherent difficulty in modelling a complex process.

There are several limitations to this study. Firstly, the number of patients involved in the study is small (recruitment of $n=17$ was completed over 3.5 years) as patients were asked to undergo a second MRI research examination. This reflects the ethical and logistical challenges of research involving children and a

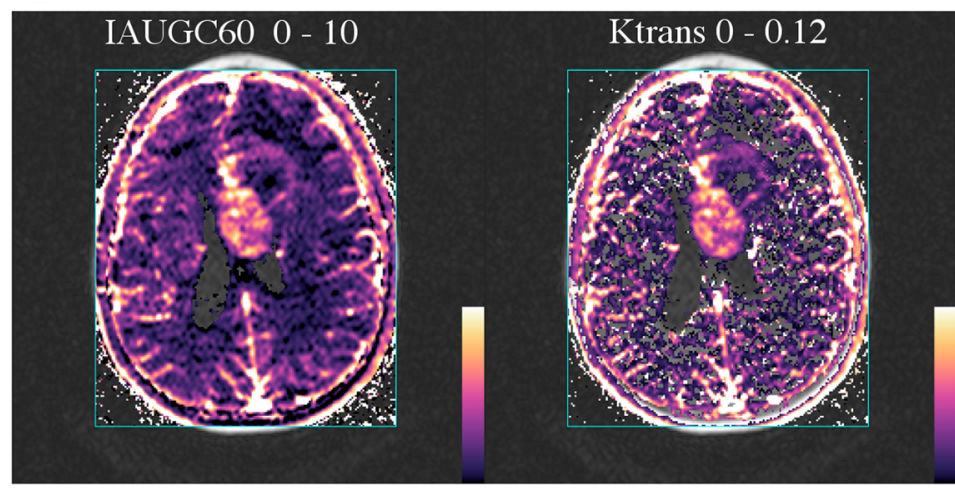

C

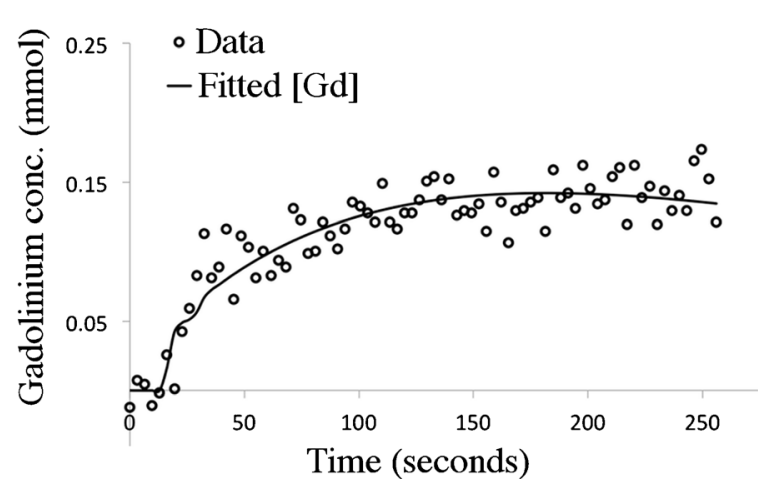

Fig. 4 Parametric maps of (a) IAUGC60 and (b) $\mathrm{K}^{\text {trans }}$ from the same patient in Fig. 2. (c) Contrast agent concentration-time course from a voxel in the tumour (circles) and the corresponding curve fit to the data (solid curve) 
a

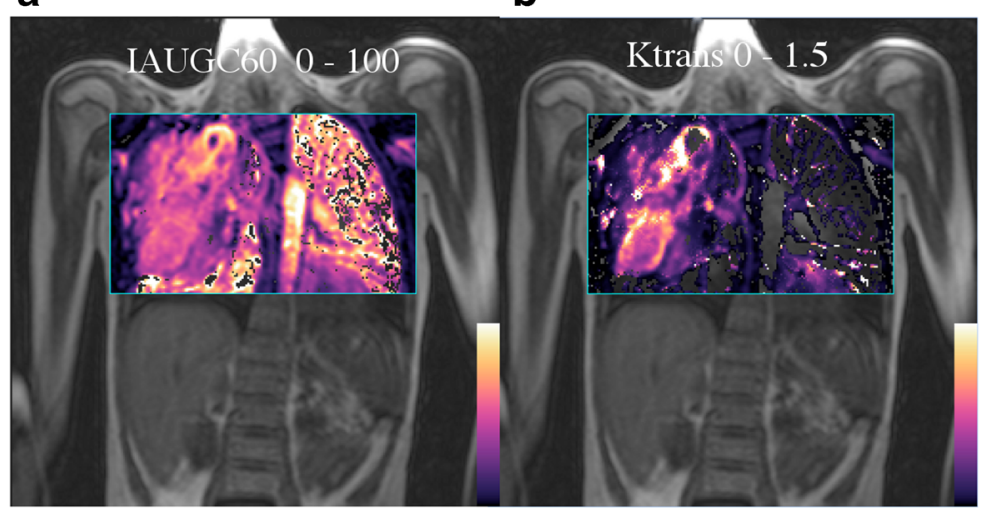

C

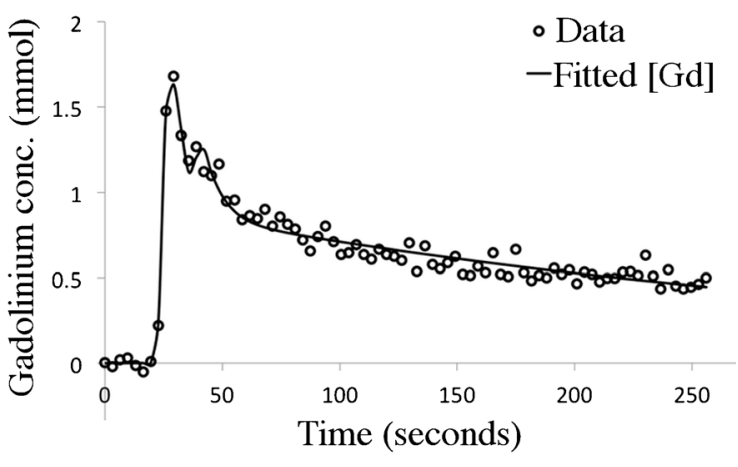

Fig. 5 Parametric maps of (a) IAUGC60 and (b) $\mathrm{K}^{\text {trans }}$ from the same patient in Fig. 3. (c) Contrast agent concentration-time course from a voxel in the tumour (circles) and the corresponding curve fit to the data (solid curve)

emphasises the importance of multi-centre collaboration to increase recruitment. The small cohort, and, therefore, limited numbers for the range of tumours represented, restricts the conclusions that can be drawn about specific tumour types, though functional parameter reproducibility is more dependent on factors arising from tumour location, imaging set-up, and acquisition parameters than from lesion type. Secondly, a population-averaged AIF for pharmacokinetic modelling of DCE-MRI data may mask differences arising from true physiological variations. Obtaining a studyspecific AIF, however, is a well-documented challenge; in our experience, it was not always possible to include an artery in the central partitions of the imaging volume. Therefore, using a population-averaged AIF was a pragmatic solution for this present study. Wang et al., have shown that it is reasonable and practical to use a limited-population-based average AIF for pharmacokinetic modelling of osteosarcoma DCE-MRI data from a large population when it is not possible to measure every individual AIF [36]. Thirdly, we have not included younger children requiring sedation or general anaesthesia for their examinations, due to the ethical challenges involved in performing repeated sedation solely for research purposes.

In conclusion, this study has demonstrated that a free-breathing protocol including diffusion-weighted and dynamic contrast-enhanced MR imaging in children aged between 6 and 15-years-old is feasible and welltolerated, and that reproducible functional parameters can be obtained. The non-invasive nature of such imaging makes this approach particularly attractive for use in a paediatric population. Findings of this pilot study will support the incorporation of DW- and DCE- MRI in paediatric phase I-II clinical trials of new molecularly targeted agents, particularly those targeting VEGF/ VEGFR and other angiogenesis pathways.
Acknowledgments The scientific guarantor of this publication is Prof. Martin Leach. The authors of this manuscript declare no relationships with any companies, whose products or services may be related to the subject matter of the article. This study has received funding by CR UK and EPSRC in association with the Medical Research Council and Department of Health (England) grant C1060/A10334, and NHS funding to the NIHR Biomedical Research Centre and the Clinical Research Facility in Imaging. Support also received for the CR UK and EPSRC Paediatric Imaging Programme C7809/ A10342. Lucas Moreno and Lynley Marshall are funded by the Oak Foundation. ADJP is supported by Cancer Research UK (programme grant C1178/A10294) - Chair in Paediatric Oncology. We acknowledge Experimental Cancer Medicine Centre (ECMC) Network funding for support to early clinical trials. No complex statistical methods were necessary for this paper. Institutional Review Board approval was obtained. Written informed consent was obtained from all subjects (patients) in this study. Methodology: prospective, experimental, performed at one institution.

Open Access This article is distributed under the terms of the Creative Commons Attribution Noncommercial License which permits any noncommercial use, distribution, and reproduction in any medium, provided the original author(s) and the source are credited.

\section{References}

1. Norris RE, Adamson PC (2012) Challenges and opportunities in childhood cancer drug development. Nat Rev Cancer 12:776782

2. Vassal G, Zwaan CM, Ashley D, Le Deley MC, Hargrave D, Blanc P et al (2013) New drugs for children and adolescents with cancer: the need for novel development pathways. Lancet Oncol 14:e117-e124

3. Boklan J (2006) Little patients, losing patience: pediatric cancer drug development. Mol Cancer Ther 5:1905-1908

4. Chang Y-C, Yu C-J, Chen C-M, Hu F-C, Hsu H-H, Tseng W-YI et al (2012) Dynamic contrast-enhanced MRI in advanced nonsmallcell lung cancer patients treated with first-line bevacizumab, gemcitabine, and cisplatin. J Magn Reson Imaging JMRI 36:387396

5. Chase DM, Sill MW, Monk BJ, Chambers MD, Darcy KM, Han ES et al (2012) Changes in tumour blood flow as measured by Dynamic Contrast-Enhanced Magnetic Resonance Imaging (DCE-MRI) may predict activity of single agent bevacizumab in recurrent epithelial 
ovarian (EOC) and primary peritoneal cancer (PPC) patients: an exploratory a. Gynecol Oncol 126:375-380

6. De Bruyne S, Van Damme N, Smeets P, Ferdinande L, Ceelen W, Mertens J et al (2012) Value of DCE-MRI and FDG-PET/CT in the prediction of response to preoperative chemotherapy with bevacizumab for colorectal liver metastases. Br J Cancer 106:19261933

7. Desar IME, Ter Voert EGW, Hambrock T, Van Asten JJ, Van Spronsen DJ, Mulders PF et al (2011) Functional MRI techniques demonstrate early vascular changes in renal cell cancer patients treated with sunitinib: a pilot study. Cancer Imaging Off Publ Int Cancer Imaging Soc 11:259-265

8. Hirashima Y, Yamada Y, Tateishi U, Kato K, Miyake M, Horita Y et al (2012) Pharmacokinetic parameters from 3-Tesla DCE-MRI as surrogate biomarkers of antitumour effects of bevacizumab plus FOLFIRI in colorectal cancer with liver metastasis. Int J Cancer 130:2359-2365

9. Jain R, Scarpace LM, Ellika S, Torcuator R, Schultz LR, Hearshen D et al (2010) Imaging response criteria for recurrent gliomas treated with bevacizumab: role of diffusion weighted imaging as an imaging biomarker. J Neuro-Oncol 96:423-431

10. Koh D-M, Blackledge M, Collins DJ, Padhani AR, Wallace T, Wilton $\mathrm{B}$ et al (2009) Reproducibility and changes in the apparent diffusion coefficients of solid tumours treated with combretastatin A4 phosphate and bevacizumab in a two-centre phase I clinical trial. Eur Radiol 19:2728-2738

11. Kummar S, Gutierrez ME, Chen A, Turkbey IB, Allen D, Horneffer YR et al (2011) Phase I trial of vandetanib and bevacizumab evaluating the VEGF and EGF signal transduction pathways in adults with solid tumours and lymphomas. Eur J Cancer 47:997-1005

12. Messiou C, Orton M, Collins DJ, Morgan VA, Mears D, Ghiorghiu D et al (2012) Advanced solid tumours treated with cediranib: comparison of dynamic contrast-enhanced MR imaging and CT as markers of vascular activity 1. Radiology 265(2): 426-36

13. Miyazaki K, Orton M, Davidson R (2012) Neuroendocrine tumour liver metastases: use of dynamic contrast-enhanced MR imaging to monitor and predict radiolabeled octreotide therapy response. Radiology 263:139-148

14. O'Connor JPB, Rose CJ, Jackson A, Watson Y, Cheung S, Maders F et al (2011) DCE-MRI biomarkers of tumour heterogeneity predict CRC liver metastasis shrinkage following bevacizumab and FOLFOX-6. Br J Cancer 105:139-145

15. Pope WB, Qiao XJ, Kim HJ, Lai A, Nghiemphu P, Xue X et al (2012) Apparent diffusion coefficient histogram analysis stratifies progression-free and overall survival in patients with recurrent GBM treated with bevacizumab: a multi-center study. J NeuroOncol 108:491-498

16. Yoo DS, Kirkpatrick JP, Craciunescu O, Broadwater G, Peterson BL, Carroll MD et al (2012) Prospective trial of synchronous bevacizumab, erlotinib, and concurrent chemoradiation in locally advanced head and neck cancer. Clin Cancer Res Off J Am Assoc Cancer Res 18:1404-1414

17. Yopp A, Schwartz L, Kemeny N (2011) Antiangiogenic therapy for primary liver cancer: correlation of changes in dynamic contrast-enhanced magnetic resonance imaging with tissue hypoxia markers and clinical response. Ann Surg Oncol 18:21922199

18. Miyazaki K, D’Arcy JA, Orton MR, Koh D-M, Collins DJ, Leach MO (2011) Improved T1 quantification using post-Gd contrast variable flip angle data. International Society of Magnetic Resonance in Medicine. p 1092

19. De Bruin P, Versluis M, Yusuf E, Reignierse M, Watt I, Van Osch M (2010) Accuracy of T1-Fitting for Pharmacokinetic Analysis of
Dynamic Contrast-Enhanced MRI. International Society of Magnetic Resonance in Medicine. p 1720

20. Miyazaki K, Orton MR, Collins DJ, d'Arcy JA, Wallace T, Moreno L et al (2013) Preliminary analysis of arterial input function derived from dynamic contrast enhanced MRI in children with cancer. International Society of Magnetic Resonance in Medicine. $\mathrm{p} 720$

21. Orton MR, Collins DJ, Messiou C, Castellano E, Tessier J, Spratt S et al (2010) A New Vascular Impulse Response Function for Modelling and Prediction with Measured Dynamic Contrast Enhanced Plasma Curves. International Society of Magnetic Resonance in Medicine. $\mathrm{p} 1726$

22. Orton MR, d'Arcy JA, Walker-Samuel S, Hawkes DJ, Atkinson D, Collins DJ et al (2008) Computationally efficient vascular input function models for quantitative kinetic modelling using DCE-MRI. Phys Med Biol 53:1225-1239

23. Parker GJM, Roberts C, Macdonald A, Buonaccorsi G, Cheung S, Buckley DL et al (2006) Experimentally-derived functional form for a population-averaged high-temporal-resolution arterial input function for dynamic contrast-enhanced MRI. Magn Reson Med Off J Soc Magn Res Med/Soc Magn Reson Med 56:9931000

24. Peet AC, Arvanitis TN, Leach MO, Waldman AD (2012) Functional imaging in adult and paediatric brain tumours. Nat Rev Clin Oncol 9: 700-711

25. Jost SC, Ackerman JW, Garbow JR, Manwaring LP, Gutmann DH, McKinstry RC (2008) Diffusion-weighted and dynamic contrast-enhanced imaging as markers of clinical behavior in children with optic pathway glioma. Pediatr Radiol 38:12931299

26. Gahr N, Darge K, Hahn G, Kreher BW, Von Buiren M, Uhl M (2011) Diffusion-weighted MRI for differentiation of neuroblastoma and ganglioneuroblastoma/ganglioneuroma. Eur J Radiol 79:443-446

27. Rodriguez Gutierrez D, Manita M, Jaspan T, Dineen R, Grundy RG, Auer DP (2013) Serial MR diffusion to predict treatment response in high-grade pediatric brain tumors: a comparison of regional and voxel-based diffusion change metrics. Neuro Oncol 15:981-989

28. Guo J, Reddick WE, Glass JO, Ji Q, Billups C, Wu J et al (2012) Dynamic contrast-enhanced magnetic resonance imaging as a prognostic factor in predicting event-free and overall survival in pediatric patients with osteosarcoma. Cancer 118: 3776-3785

29. Navid F, Baker SD, McCarville MB, Stewart CF, Billups C, $\mathrm{Wu} \mathrm{J}$ et al (2013) Phase I and clinical pharmacology study of bevacizumab, sorafenib, and low-dose cyclophosphamide in children and young adults with refractory/recurrent solid tumors. Clin Cancer Res Off J Am Assoc Cancer Res 19:236246

30. Nathan P, Zweifel M, Padhani AR, Koh D-M, Ng M, Collins DJ et al (2012) Phase I trial of combretastatin A4 phosphate (CA4P) in combination with bevacizumab in patients with advanced cancer. Clin Cancer Res Off J Am Assoc Cancer Res 18:3428-3439

31. Jamin Y, Tucker E, Poon E, Popov S (2013) Evaluation of clinically translatable MR imaging biomarkers of therapeutic response in the TH-MYCN transgenic mouse model of neuroblastoma. Radiol 266: $130-140$

32. O'Connor JPB, Carano RAD, Clamp AR, Ross J, Ho CCK, Jackson A et al (2009) Quantifying antivascular effects of monoclonal antibodies to vascular endothelial growth factor: insights from imaging. Clin Cancer Res Off J Am Assoc Cancer Res 15: 6674-6682

33. Leach MO, Brindle KM, Evelhoch JL, Griffiths JR, Horsman MR, Jackson A et al (2005) The assessment of antiangiogenic and 
antivascular therapies in early-stage clinical trials using magnetic resonance imaging: issues and recommendations. $\mathrm{Br} \mathrm{J}$ Cancer 92: $1599-1610$

34. Leach MO, Morgan B, Tofts PS, Buckley DL, Huang W, Horsfield MA et al (2012) Imaging vascular function for early stage clinical trials using dynamic contrast-enhanced magnetic resonance imaging. Eur Radiol 22:1451-1464
35. Quantitative Imaging Biomarkers Alliance. Profile: DCE MRI Quantification Version 1.6 (2011)

36. Wang Y, Huang W, Panicek DM, Schwartz LH, Koutcher JA (2008) Feasibility of using limited-population-based arterial input function for pharmacokinetic modeling of osteosarcoma dynamic contrastenhanced MRI data. Magn Reson Med Off J Soc Magn Reson Med/Soc Magn Reson Med 59:1183-1189 